1-S09-3 Symposium9

\title{
Efforts by The Japanese Association for Experimental Animal Technologists (JAEAT) : Scheduled technical training courses and their future development
}

\author{
Norio Yata $^{1,2}$
}

${ }^{1}$ Advanced Science Research Center, Okayama Univ., ${ }^{2}$ Director of The Japanese Association for Experimental Animal Technologists (JAEAT)

We, laboratory animal technicians, are always the closest to laboratory animals. We always have practiced breeding management, pre- and post-operative care, experimental treatment etc. by making the best use of our knowledge and techniques. That bring accurate experimental results. It is our significance.

"Skill" is individual and the only way to earn it is by training. On the other hand, "technique" is objective and can be transmitted by words and letters. Our goal is not to acquire skill called "SHOKUNINWAZA" in Japanese, but it is to make it universal as an objective technique that anyone can practice. The training course plays the important role to attain our goal.

The Japanese Association for Experimental Animal Technologists (JAEAT) was founded in 1966. We organize annual meeting, lecture and technical training course. We, JAEAT KANSAI branch, actively hold the training courses 4 times a year. In every training, one-on-one instruction is highly esteemed.

We created DVD video for basic experimental techniques. And graphical chart was published 2 years ago. Which were laminated, to bring in our bloody workspace. We can use them anytime, anywhere.

Technology is only meaningful if passed down to the next generation. Even failure is a valuable experience there. Whether it worked or not, everything is not a personal experience. From there we take out the universal contents and pass on them to the next generation based on animal welfare. It is our mission and we have no doubt.

In the presentation, I introduce our efforts to pass on the laboratory animal technique to next generation. 